\title{
Feasibility of Merging Sacroiliac Fusion Computed Tomography Preoperative Planning to Original Computed Tomography Scans
}

\author{
Matthew D. Alvin ${ }^{1,2}$, Mu Yang ${ }^{2}$ and Michael MacMillan, ${ }^{2, *}$ \\ ${ }^{I}$ Case Western Reserve University School of Medicine, Cleveland, Ohio, USA \\ ${ }^{2}$ University of Florida Orthopaedic and Sports Medicine Institute, Gainesville, Florida, USA
}

\begin{abstract}
Background: Spinal merging has been done with preoperative CT scans merged with fluoroscopic imaging. However, preoperative and intraoperative CT scans have not been merged for the purposes of transferring a plan. If spine surgeons can create the optimal surgical pathway before surgery and then merge the pre-planned pathway onto the real patient CT scan in the operating room, the result will be efficiency and better time management for the spine surgeon. Our objective was to analyze the feasibility of merging computed tomography (CT) preplanned with sacroiliac fusion iliosacral screw trajectories with the original CT scans.

Methods: Twenty-seven patients were identified from the institutional Faculty Group Practice who had undergone CT scans in the pelvic region. The pelvic CT scans were transferred to Medtronic's StealthStation ${ }^{\circledR}$, which allowed for analysis in coronal, sagittal, and axial sections. Three trajectories were mapped out, representing three working pathways for fixation. The position of the trajectories was localized by measuring their distance from anatomic landmarks. The CT scan with the planned trajectories was merged with the original CT scan. The same measurements made pre-merge were performed post-merge to check for the accuracy of the merging process.
\end{abstract}

Results: Post-merge measurements were nearly identical to pre-merge measurements, with a maximum difference of \pm 0.1 $\mathrm{mm}$. No significant differences existed between pre-merge and post-merge measurements for any of the trajectories

Conclusion: The merging of preplanned trajectory CT scans with intraoperative CT scans is a highly accurate technique.

Clinical relevance: If this technique can be replicated by merging actual pre-operative CT scans with actual intraoperative scans, it could save time and expense. This would benefit the patient because time under anesthesia would be reduced.

Keywords: Sacroiliac fusion, virtual screw, CT merge, iliosacral screw, pelvic injury.

\section{INTRODUCTION}

Spine surgeons are seeking safer and more effective procedures which will allow the patient to experience less pain and recover more quickly. Sacroiliac joint fusion is a common procedure performed for patients with dislocations or fractures. Specifically, about $15 \%$ to $30 \%$ of patients with low back pain have sacroiliac joint dysfunction $[1,2]$. In the past, sacroiliac joint fusion commonly involved open exposure of the joint, decortication, bone grafting and, finally, fixation. Minimally invasive approaches have become much more popular recently in reducing the morbidity associated with open approaches. The minimallyinvasive approach of choice available for most surgeons has been a combination of biplanar fluoroscopic imaging and iliosacral screws [2]. However, fluoroscopy has its disadvantages including impaired visualization of the

*Address correspondence to this author at the Orthopaedics and Sports Medicine Institute, University of Florida - Spine Division, P.O. Box 112727, Gainesville, Florida 32601, Tel: 352-273-7434; Fax: 352273 7388; E-mail:macmim@ortho.ufl.edu trajectory due to obesity, bowel gas, or contrast media and the level of technical skills required [2,3]. Computed tomography (CT) scans present a less technically demanding option for trajectory mapping and have been studied extensively [3-10].

To improve visualization for sacroiliac fixation, a 3-D fluoroscopic image of the patient's pelvis is performed and transferred to the StealthStation ${ }^{\circledR}$ Navigation station (Medtronic, Inc., Memphis, TN). Utilizing the cross sectional images, the surgeon presently plans trajectory in the operating room which adds time and effort to the procedure. If it were possible to map out the trajectory prior to the day of the surgery on an available diagnostic CT, surgery time would be reduced. Prior spinal planning has been done with preoperative CT scans merged with intraoperative fluoroscopic imaging [3]. However, preoperative CT scan merging with the intraoperative CT scan for the purposes of transferring a plan to date has only been done with cranial software - not in the spine. The intent of the present study is to establish grounds for further study on the merging process. While imaging is used for intracranial and spine procedures, the process of merging a preplanned 


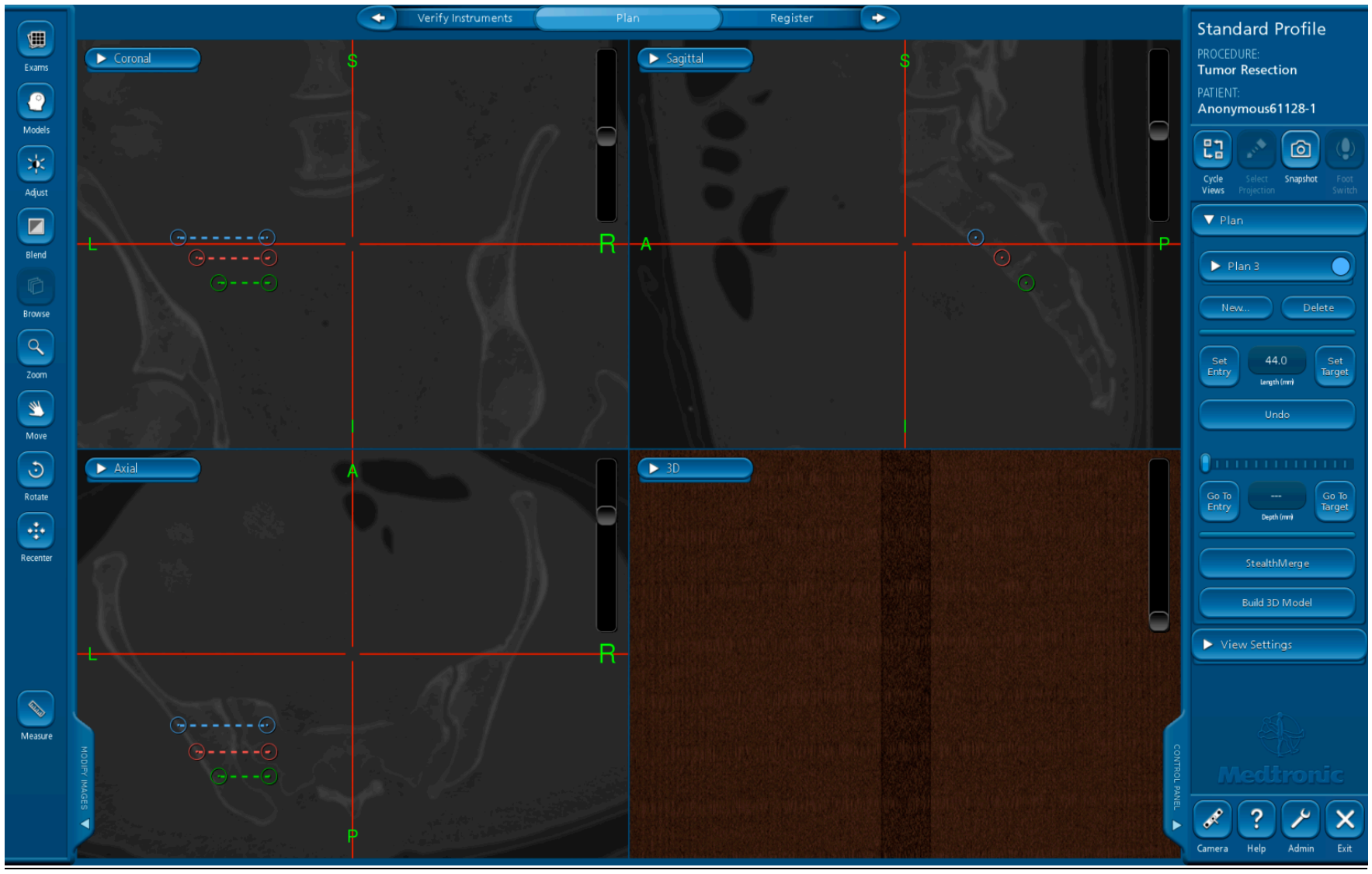

Fig. (1). User interface of StealthStation ${ }^{\circledR}$ Navigation station (Medtronic, Inc., Memphis, TN). Three panels (upper left, upper right, bottom left) show coronal, sagittal, and axial CT scans, respectively. Three hypothetical screws (blue, red, green) were drawn with entry and target points connected by dashed lines.

image with an intraoperative image has only been studied for intracranial procedures. In the present study, we analyzed the feasibility of merging a pre-planned preoperative pelvic CT scan with the original pelvic CT scan on Medtronic's StealthStation ${ }^{\circledR}$ Navigation system. Our hypothesis was that the merging process would be highly accurate and warrant further clinical investigation and consideration for application to the spine surgeon's practice.

\section{METHODS}

\section{Demographics}

After approval by the local Institutional Review Board, a list was generated of patients who had undergone CT scans in the pelvic region between March 2008 and April 2012. Only patients with no anatomical abnormalities were included. Patient demographic data (gender, age) was deidentified prior to acquisition.

\section{Virtual Trajectory Analysis}

The pelvic CT scans were transferred to Medtronic's StealthStation $^{\circledR}$, which allowed for analysis in coronal, sagittal, and axial sections (Fig. 1). Three trajectories were mapped out, representing three working channels for fusion instrumentation. The trajectories were separated out to be as equidistant from each other as possible. The entry point for each trajectory was chosen as the outermost feasible portion of the ilium, whereas the target point was chosen as the innermost point of the sacral ala on right and left sides, respectively. Once the three trajectories were mapped out, multiple measurements were taken, including the distance from the entry point to the target point and the distance from the entry point and target point to three landmarks: the coccyx, the L5 spinous process, and the sacral promontory. Figs. (2 and $\mathbf{3}$ ) are provided for an understanding of the exact measurements performed.

After taking the pre-merge measurements, the CT scan with the planned trajectories was saved to a DVD-ROM. The original CT scan without the planned trajectories was then reopened and merged with the $\mathrm{CT}$ scan with the planned trajectories. A completely successful transfer of the plans in the merging process was defined as the plans appearing in the exact same location (via measurements) on the merged scan as they appeared on the original planned trajectory scan. Thus, the same measurements made pre-merge were performed post-merge to check for the accuracy of the margining process.

\section{STATISTICAL METHODS}

Pre- and post-merge data sets were compared using the Student's t-test. All p-values $\leq 0.05$ were considered statistically significant.

\section{RESULTS}

Twenty-seven patient CT scans were analyzed based on the inclusion criteria for the study (Table 1). The patient 


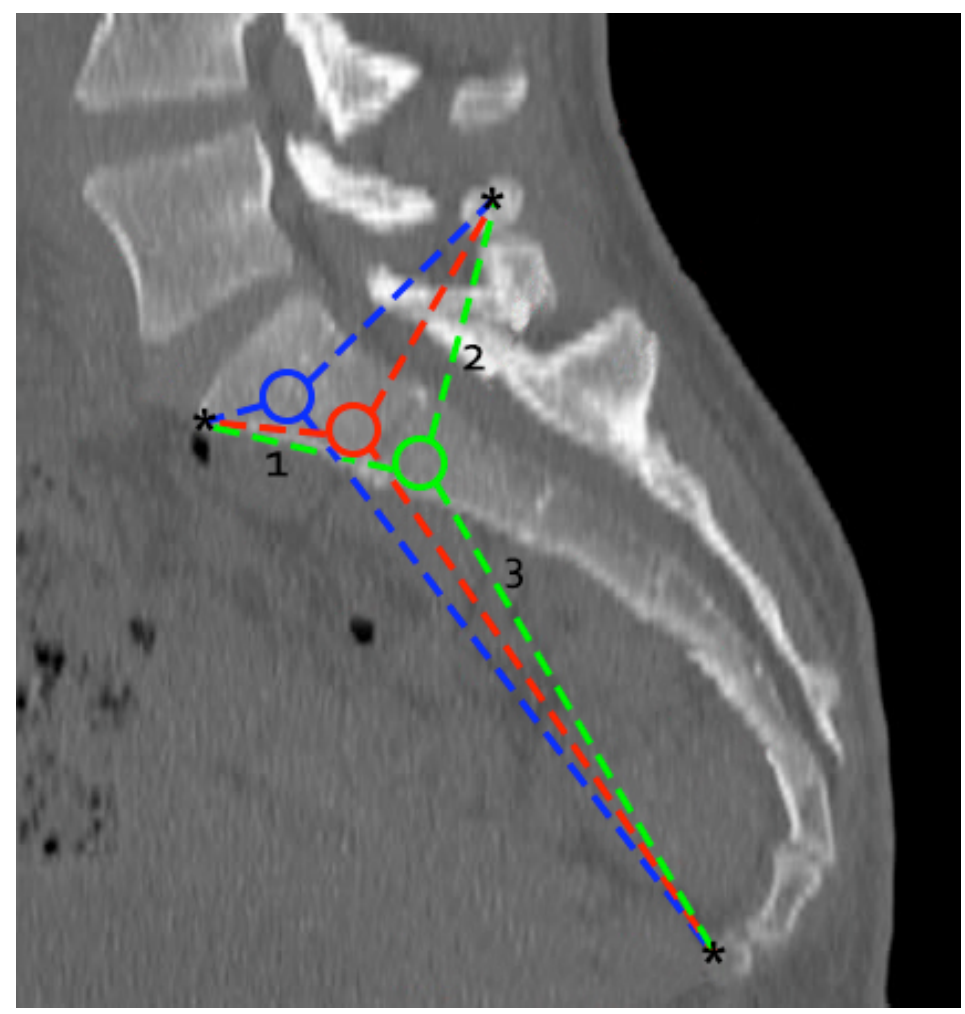

Fig. (2). Blue, red, and green circles represent three different hypothetical screws involved in sacroiliac fusion. The three black asterisks identify the sacral promontory, L5 spinous process, and coccyx. The dashed lines $(\# 1,2,3)$ represent the measurements taken from each screw to the sacral promontory (\#1), L5 spinous process (\#2), and coccyx (\#3).

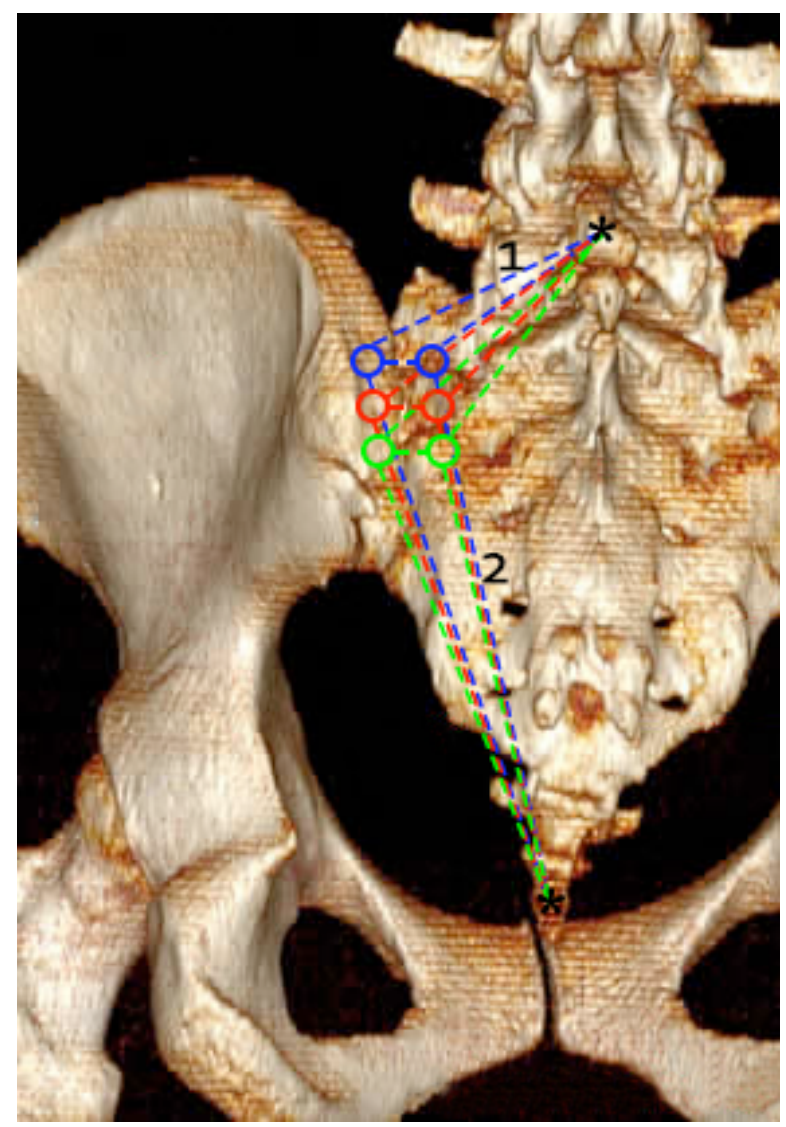

Fig. (3). Blue, red, and green circles represent three different hypothetical screws involved in sacroiliac fusion. The two sets of circles represent entry points and target points, respectively. The two black asterisks identify the L5 spinous process and coccyx. The dashed lines $(\# 1,2)$ represent the measurements taken from each screw to the L5 spinous process (\#1) and coccyx $(\# 2)$. 
Table 1. Demographics

\begin{tabular}{|c|c|}
\hline Demographic & Patient Cohort \\
\hline \hline $\mathrm{n}$ & 27 \\
\hline Age & $42.5 \pm 17.6$ \\
\hline Gender & $4(15 \%)$ \\
Male & $23(85 \%)$ \\
Female & \\
\hline
\end{tabular}

Table 2. Trajectory \#1

\begin{tabular}{|c|c|c|c|}
\hline & Pre-Merge (mm) & Post-Merge (mm) & p-value \\
\hline $\mathrm{L}-\mathrm{EP} 1$ to TP1 & $40.7[40.5,40.9]$ & $40.7[40.4,41.0]$ & 0.9 \\
\hline $\mathrm{R} 1$ - EP (C) & $135.9[135.5,136.4]$ & $135.9[135.3,136.4]$ & 0.9 \\
\hline R1 - EP (S) & $95.8[95.1,96.6]$ & $95.8[95.1,96.5]$ & 0.9 \\
\hline $\mathrm{R} 1-\mathrm{TP}(\mathrm{C})$ & $111.3[110.8,111.8]$ & $111.3[110.7,111.8]$ & 0.9 \\
\hline $\mathrm{R} 1-\mathrm{TP}(\mathrm{S})$ & $61.7[61.1,62.3]$ & $61.6[61.0,62.3]$ & 0.9 \\
\hline $\mathrm{R} 1$ - TP (P) & $48.7[48.2,49.2]$ & $48.7[48.2,49.2]$ & 0.9 \\
\hline L1 - EP (C) & $133.4[132.9,133.9]$ & $133.4[132.9,133.9]$ & 0.9 \\
\hline $\mathrm{L} 1$ - TP (S) & $60.7[60.0,61.4]$ & $60.7[60.0,61.4]$ & 0.9 \\
\hline $\mathrm{L} 1-\mathrm{TP}(\mathrm{P})$ & $48.9[48.4,49.4]$ & $48.8[48.4,49.3]$ & 0.9 \\
\hline
\end{tabular}

Mean and 95\% confidence interval are reported; EP = Entry Point; TP = Target Point; R = Right; $\mathrm{L}=$ Left; $\mathrm{C}=$ Coccyx; $\mathrm{S}=\mathrm{L} 5$ spinous process; $\mathrm{P}=$ Sacral Promontory.

Table 3. Trajectory \#2

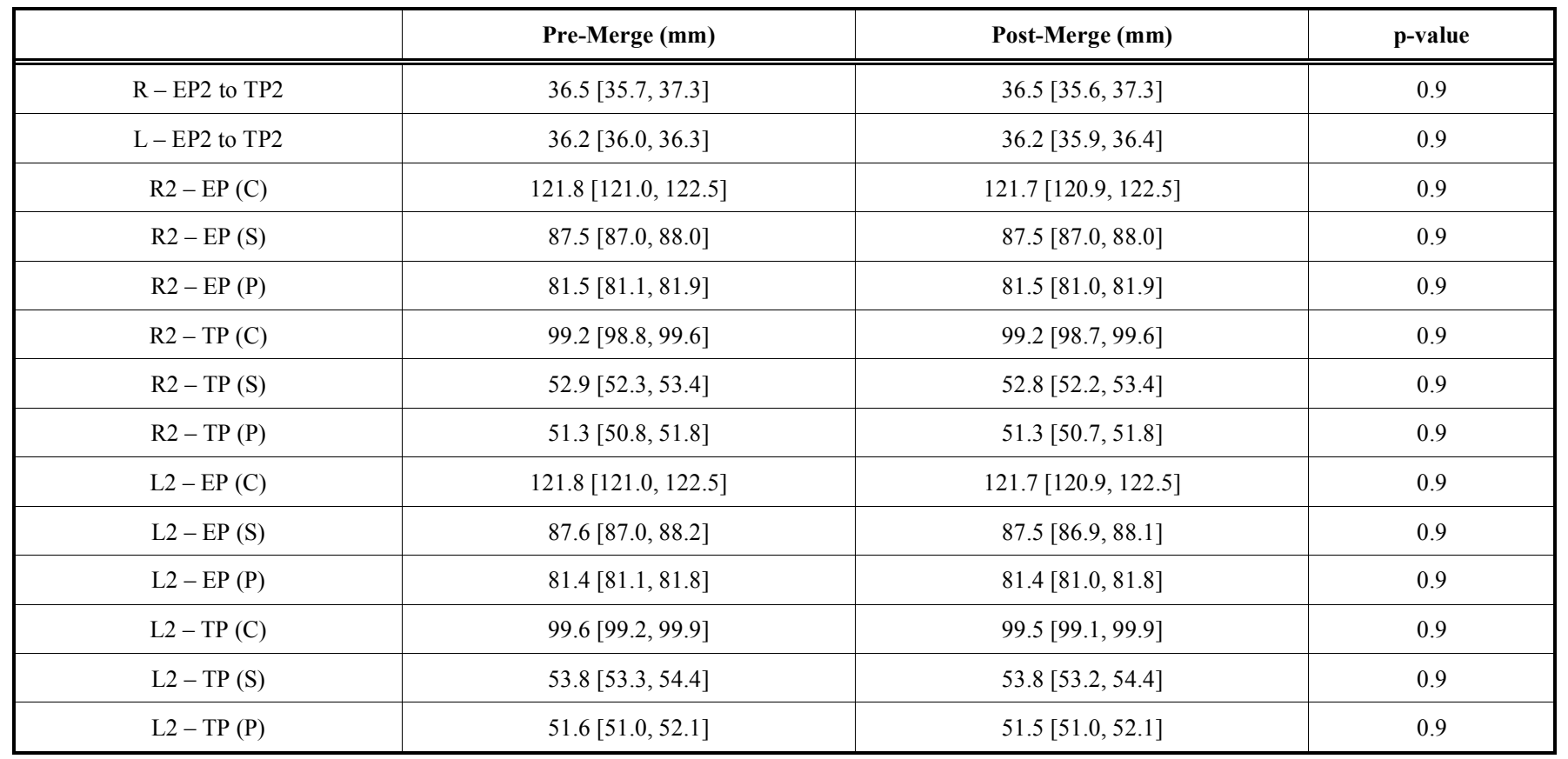

Mean and 95\% confidence interval are reported; EP = Entry Point; TP = Target Point; $\mathrm{R}=$ Right; $\mathrm{L}=$ Left; $\mathrm{C}=$ Coccyx; $\mathrm{S}=\mathrm{L} 5$ spinous process; $\mathrm{P}=$ Sacral Promontory. 
Table 4. Trajectory \#3 and Distance Between Trajectories

\begin{tabular}{|c|c|c|c|}
\hline & Pre-Merge (mm) & Post-Merge (mm) & p-value \\
\hline $\mathrm{R}-\mathrm{EP} 3$ to TP3 & $24.3[24.0,24.6]$ & $24.2[23.9,24.6]$ & 0.9 \\
\hline $\mathrm{L}-\mathrm{EP} 3$ to TP3 & $24.1[24.0,24.2]$ & $24.1[24.0,24.2]$ & 0.9 \\
\hline R3 - EP (C) & $105.5[105.0,105.9]$ & $105.4[105.0,105.9]$ & 0.9 \\
\hline R3 - EP (S) & $68.7[68.2,69.3]$ & $68.7[68.1,69.3]$ & 0.9 \\
\hline $\mathrm{R} 3-\mathrm{EP}(\mathrm{P})$ & $76.3[75.8,76.9]$ & $76.3[75.7,76.8]$ & 0.9 \\
\hline R3 - TP (C) & $95.8[95.4,96.2]$ & $95.7[95.3,96.1]$ & 0.9 \\
\hline R3 - TP (S) & $42.4[42.0,42.7]$ & $42.3[41.9,42.8]$ & 0.9 \\
\hline R3 - TP (P) & $57.5[57.0,58.1]$ & $57.5[56.8,58.2]$ & 0.9 \\
\hline L3 - EP (C) & $106.0[105.6,106.4]$ & $106.0[105.5,106.4]$ & 0.9 \\
\hline L3 - EP (S) & $69.1[68.6,69.7]$ & $69.1[68.5,69.7]$ & 0.9 \\
\hline L3 - EP (P) & $77.3[76.8,77.8]$ & $77.3[76.8,77.7]$ & 0.9 \\
\hline L3 - TP (C) & $96.5[96.0,97.0]$ & $96.5[95.9,97.0]$ & 0.9 \\
\hline L3 - TP (S) & $42.9[42.4,43.4]$ & $42.8[42.3,43.3]$ & 0.9 \\
\hline L3 - TP (P) & $57.3[56.1,58.4]$ & $57.2[56.1,58.4]$ & 1.0 \\
\hline $\mathrm{R}-\mathrm{T} 1$ and $\mathrm{T} 2$ & $16.0[15.9,16.0]$ & $16.0[15.8,16.1]$ & 0.6 \\
\hline $\mathrm{R}-\mathrm{T} 1$ and $\mathrm{T} 3$ & $33.1[32.9,33.2]$ & $33.0[32.8,33.2]$ & 0.7 \\
\hline $\mathrm{R}-\mathrm{T} 2$ and $\mathrm{T} 3$ & $17.2[17.1,17.3]$ & $17.2[17.0,17.4]$ & 0.6 \\
\hline $\mathrm{L}-\mathrm{T} 1$ and $\mathrm{T} 2$ & $15.5[15.3,15.7]$ & $15.5[15.2,15.8]$ & 0.6 \\
\hline $\mathrm{L}-\mathrm{T} 1$ and $\mathrm{T} 3$ & $30.2[29.9,30.7]$ & $30.3[30.0,30.6]$ & 0.7 \\
\hline $\mathrm{L}-\mathrm{T} 2$ and $\mathrm{T} 3$ & $15.2[14.8,15.7]$ & $15.3[15.1,15.5]$ & 0.6 \\
\hline
\end{tabular}

Mean and 95\% confidence interval are reported; $\mathrm{EP}=$ Entry Point; $\mathrm{TP}=$ Target Point; $\mathrm{R}=$ Right $\mathrm{L}=\mathrm{Left}$ C $=$ Coccyx; $\mathrm{S}=\mathrm{L} 5$ spinous process; $\mathrm{P}=\mathrm{Sacral}$ Promontory; $\mathrm{T}=$ Trajectory where $\mathrm{T} 1$ and $\mathrm{T} 2$ represents the distance between the trajectories.

population average age was $42.5 \pm 17.6$ years and $85 \%$ $(23 / 27)$ were females with $15 \%(4 / 27)$ males.

All pre-merge and post-merge trajectory measurements are listed in Tables $\mathbf{2}, \mathbf{3}$ and $\mathbf{4}$. No significant differences existed between pre-merge and post-merge measurements for any of the trajectories. No significant differences existed between males and females or by age for any of the trajectories.

\section{DISCUSSION}

In the past two decades, many investigations for improved navigation systems for iliosacral screw fixation in sacroiliac fusion procedures have been conducted. In 1994, Ebraheim et al. [4] analyzed a cohort of 19 patients with posterior pelvic fractures undergoing sacroiliac fusion using CT-scan guidance intraoperatively. High accuracy was achieved and blood loss and associated complications were minimal. The authors stated that CT scans provided a very precise visualization of iliosacral screw placement. In 1998, Barrick et al. [3] evaluated the efficacy of a computerassisted CT scan guided system for iliosacral screw fixation in cadavers with posterior pelvic ring injuries. High success rates, defined as the presence of each iliosacral screw fully inside bone, were seen in the study, leading the authors to recommend clinical trials to be undertaken. Kahler and
Mallick [5] further studied this navigation system while Tonetti et al. [6] reported on a similar navigation system using CT scans and ultrasound.

In 2001, Blake-Toker et al. [7] found that CT-guided fixation of unstable pelvic fractures minimized blood loss, had minimal complications, and allowed earlier mobilization of patients $(n=20)$ in comparison to open reduction techniques. In 2010, Klauser et al. [8] evaluated an image fusion guidance technology in five cadavers and seven patients for sacroiliac joint injection. Confirmation of high accuracy was seen on CT scans. No complications occurred intraoperatively. Thus, the authors concluded it was a feasible technique. In 2012, Peng et al. [9] analyzed an iliosacral screw integrated navigation system with CT scanning in patients $(n=13)$ who presented with pelvic fractures with posterior ring disruption. The authors found high accuracy, defined as lack of penetration or encroachment of a foramen/nerve or the spinal cord, with no injuries in any of the 13 cases. However, the effective radiation dose was higher than fluoroscopic-assisted techniques. Zhanle et al. [10] conducted a similar study, but used a thermoplastic membrane navigation system $(n=13)$ for patients with sacroiliac joint fractures or dislocations. They also utilized a control group $(n=13)$ receiving fluoroscopic guidance and found that their thermoplastic 
navigation system provided significantly reduced operative time and blood loss with high accuracy.

In the present study, we retrospectively assessed pelvic CT scans for the feasibility of merging the preplanned trajectory CT scans for 27 patients with the original scans. The results show the high accuracy of the merging process and warrant further investigation of this technique for application to clinical practice. This study represents the first to analyze the feasibility of merging CT scans for spinal procedures. It is essential to understand that this study analyzes merging preplanned CT scans with intraoperative real-time CT scans. It is well-known that intraoperative imaging guidance is used routinely for both intracranial and spinal procedures. However, the merging of an already planned out surgery on a preoperative CT scan with a realtime intraoperative CT scan has not been studied in the spine. In addition, the patient sample size is larger than most previous studies in the literature reporting on CT scan applicability for sacroiliac fusion.

We recognize several limitations of the study that need to be addressed. First, $85 \%$ of the patients in this study were females. Second, variations in sacral anatomy pose an issue in generalizing the results of any image-guided study to the general population with regards to mapping trajectories. Third, errors from CT imaging, measurement error, and human error must be taken into account. In our study, the pre-operative plans were merged onto the exact same CT data set. This represents the most ideal circumstances for merging to be successful and certainly skewed the data for accuracy. Given the high accuracy of the merging process as seen in this study, further research is warranted. The success of this method using different CT data sets and subsequently in clinical trials needs to be assessed.

The merging of preplanned trajectory CT scans with intraoperative CT scans would save the spine surgeon time and effort and contribute to reduced operative time. This would result in savings in health care dollars and the advantage to the patient of less time under anesthesia.

\section{CONFLICT OF INTEREST}

The authors confirm that this article content has no conflicts of interest.

\section{ACKNOWLEDGEMENTS}

The authors would like to thank Sonya Tang for her assistance with the IRB protocol preparation, Joanne Clarke for her review of the manuscript and administrative assistance, and Mike Truchon for graphic design of the Figures in this manuscript.

\section{REFERENCES}

[1] DePalma MJ, Kecthum JM, Saullo T. What is the source of chronic low back pain and does age play a role? Pain Med 2011; 12: 22433.

[2] Phillips FM, Garfin SR. Minimally invasive sacroiliac joint fusion. Orthopedics 2011; 34: 600-1.

[3] Barrick EF, O'Mara JW, Lane HE 3rd. Iliosacral screw insertion using computer-assisted CT image guidance: a laboratory study. Comput Aided Surg 1998; 3: 289-96.

[4] Ebraheim NA, Coombs R, Jackson WT, Rusin JJ. Percutaneous computed tomography-guided stabilization of posterior pelvic fractures. Clin Orthop Relat Res 1994; 307: 222-8.

[5] Kahler DM, Mallik K. Early experience with a computer assisted technique for iliosacral screw placement in posterior pelvic ring disruptions. Syllabus of CAOS/USA 1998, Pittsburgh. pp. 180-182.

[6] Tonetti J, Carrat L, Lavalle'e S, et al. Percutaneous iliosacral screw placement using image guided techniques. Clin Orthop Relat Res 1998; 354: 103-10.

[7] Blake-Toker AM, Hawkins L, Nadalo L, et al. CT Guided percutaneous fixation of sacroiliac fractures in trauma patients. J Trauma 2001; 51: 1117-21.

[8] Klauser AS, De Zordo T, Feuchtner GM, et al. Fusion of real-time US with CT images to guide sacroiliac joint injection in vitro and in vivo. Radiology 2010; 256: 547-54.

[9] Peng KT, Li YY, Hsu WH, et al. Intraoperative computed tomography with integrated navigation in percutaneous iliosacral screwing. Injury 2013; 44: 203-8.

[10] Zheng Z, Zhang Y, Hou Z, et al. The application of a computerassisted thermoplastic membrane navigation system in screw fixation of the sacroiliac joint - a clinical study. Injury 2012; 43: 495-9. 